\title{
Requerimientos de aplicación de una estrategia digital dual de comercialización por una empresa PyME dedicada a la venta de café
}

\author{
Andrea González Acevedoํㅣㄹ Juan Benito Vela Reyna² y Eduardo Sánchez López ${ }^{3}$ \\ ${ }^{1}$ Universidad Autónoma de Baja California, Andrea.gonzalez.acevedo@uabc.edu.mx.Rio Nuevo y eje \\ central s/n col Agualeguas (+52) 6865823377 \\ ${ }^{2}$ Universidad Autónoma de Baja California, jbenitovela@uabc.edu.mx. Rio Nuevo y eje central s/ $\mathrm{n}$ col \\ Agualeguas (+52) 6865823377 \\ ${ }^{3}$ Universidad Autónoma de Baja California, eduardosanchez@uabc.edu.mx. Rio Nuevo y eje central s/n col \\ Agualeguas (+52) 6865823377
}

Información del artículo revisado por pares|
Fecha de aceptación: junio-2021
Fecha de publicación en línea: diciembre-2021
DOI: https://doi.org/10.29105/vtga7.2-39

\section{Resumen}

La evolución en el mundo, el crecimiento de ciudad, la forma de consumo en el mundo ha cambiado y, tanto usuarios como empresas, necesitan establecer nuevos mecanismos de comunicación y comercialización. En la actualidad 6 de cada 10 empresa vende por internet, las empresas saben que tener una presencia digital es obligatorio en la actualidad, de forma introductoria lo hacen por redes sociales, en ellas comparten imágenes, información de sus productos o servicios, o incluso precios. Aunque los canales físicos y las bases digitales propias son importantes, las PYMES saben de la fuerza de venta que significan multiplataformas como Amazon, mercado libre, e-bay por citar algunos. La importancia crece diariamente y el público se anima a comprar por medios digitales. Al final, lo que buscan es el aumento de venta en general. El presente trabajo, describe las recomendaciones que una empresa dedicada a la comercialización de una marca de café en la ciudad de Mexicali Baja California, comience una alternativa en ventas en un entorno digital.

Palabras clave: PYME, plataforma, digital, comercialización, estrategia

\begin{abstract}
The evolution in the world, the growth of the city, the way of consumption in the world has changed and both users and companies need to establish new communication and marketing mechanisms. Currently 6 out of 10 companies sells online, companies know that having a digital presence is a mandatory today, initially y do it through social networks, where they share images, information about their products or services, or even prices.

Although physical channels and their own digital bases are important, SMEs know of the sales force that multiplatform such as Amazon, Mercado Libre, e-bay, to name a few. The importance grows daily and the public is encouraged to buy digitally. In the end, what they are looking for is an increase in sales in general. This work describes the recommendations that a company dedicated to the commercialization of a coffee brand in the city of Mexicali Baja California, start an alternative in sales in a digital environment.
\end{abstract}

Keywords: SMEs, plataform, digital, commercialization, strategy 


\section{OBJETIVO GENERAL}

Determinar las necesidades mercadológicas y técnicas requeridas por una empresa PYME dedicada a la venta de café para la aplicación de una estrategia comercial digital dual que considere ventas directas minoristas y ventas directas físicas a mayoristas mediante plataformas digitales.

\section{OBJETIVO PARTICULAR}

Determinar las características del negocio como base para la ampliación y mejora de la estrategia comercial que aplica.

Definir las características del mercado hacia el cual se orientará la nueva estrategia comercial.

\section{INTRODUCCION}

\section{Comercio electrónico y PYMES}

En una publicación sobre la transformación digital en las pequeñas y medianas empresas, la Organización para la Cooperación y el Desarrollo Económicos, (OCDE 2021), confirma que un factor importante de crecimiento en las PYMES es la "migración a lo digital", como parte de una transición digital de economías y sociedades como fortaleza ante la pandemia Covid-19.

Según este organismo la OCDE (2021), esto ha abierto la posibilidad de nuevos formatos de ventas y facilidades de acceso a un sinfín de conexiones digitales entre empresas y público, lo que hace que derive en una expansión y crecimiento de las PYMES. De igual forma, se centra en los beneficios que esto puede tener en las empresas, desde la disminución de costos operativos, pasando por el casi obvio aumento de clientes, hasta la generación de una economía de escala, capitalizando los efectos de las nuevas conexiones con clientes y empresas.

Por otro lado, el estudio también refleja los riesgos y los desafíos nuevos de las pymes en ese sentido. Por ejemplo, la falta de conocimiento digital, la adaptación de un modelo de negocio apto para la nueva situación, en general, el conocimiento apropiado para la operación de una empresa con las nuevas características.

Según un estudio sobre venta online en PYMES 2020 (AMVO, 2020), en México las ventas en línea las hacen 6 de cada 10 PYMES, con ello, muestra un cremento del $94.6 \%$ comparado con 2019. Ha sido, debido a la situación mundial actual, que 2 de cada 10 PYMES vende de esta forma. Esto no resta importancia del crecimiento de estos nuevos canales, sino que, se toma en cuenta como acelerador del mismo. Las empresas tienen diferentes opciones para generar venta, una de ellas es mediante sus páginas web, sin embargo, las más conocidas para la venda con las grandes plataformas multi-categorías, como, por ejemplo, mercado libre, amazon, ebay, por citar algunas.

\section{Las ventajas competitivas de las ventas en línea}

Como se ha mencionado anteriormente, algunas de las ventajas primordiales que hacen los organismos internacionales, respecto a las ventas online $\mathrm{o}$ multiplataformas, es precisamente el ahorro en ciertos gastos operativos, lo que permite a la pyme, aumentar ese margen de ganancia que, de otro modo, se vería muy sacrificado. (OECD, 2021)

Según la publicación, Marketing digital para PYMES (Guru, n.d.) una de las ventajas en el aumento de la presencia de marca, informa que, entre mayor visitas tenga el sitio web, obtendrá un mayor lugar en los resultados de búsqueda de Google. De igual forma, se conecta mejor y mayormente de los clientes por medio de las redes sociales, ya que se puede interactuar de forma directa $y$ personalizada. Mencionan, algo importante sobre la posibilidad de atraer nuevos clientes, mediante imágenes, infografías, videos etc. Con ello, aumentar las ventas de forma directa.

Según información de Interactive Advertising Bureau (IAB México 2019) las principales razones por las que una persona compra de forma virtual, es debido a que recibe sus compras a domicilio con una valoración del 
$58 \%$ por el usuario. Otro punto importante es que les ahorra tiempo debido a que no deben trasladarse, hacer filas etc. Por otro lado, el $48 \%$ de los usuarios comentó que encuentra mayores promociones y descuentos que en las tiendas físicas.

Por otro lado, el estudio arroja que los atributos buscados por los usuarios en las páginas web que motivan la compra es precisamente la facilidad de la navegación en el mismo, seguido del diseño de la página, la funcionalidad, claridad en garantías y tiempos de entrega y por último el proceso de pago.

Todo lo anterior, se conjunta de forma tal, que permite a los usuarios una comodidad y tranquilidad de que sus productos llegarán como han sido presentados en tiempo y forma.

El café, al tratarse de un producto de fácil manejo y acceso, es muy bien aceptado por los consumidores en general. En cuanto a las referencias cualitativas del producto, la preferencia del grano va en un consumo de café soluble y molido. Con la referencia anterior, el consumo se espera crezca un promedio de $2.5 \%$, lo que sitúa un consumo per cápita de 1.5 kilos. (Euromonitor 2017).

Según una publicación de la revista Forbes, (2020) empresas multinacionales como Nespresso, aumentó de cuatro a cinco veces durante el año 2020 debido al consumo de café en los hogares por cuestiones de la contingencia que se vive. De igual forma, detectaron que los usuarios regresaron a realizar una segunda compra de este producto. Si se toma como referencia la información anterior, se puede observar que las ventas digitales son una excelente opción para las ventas en las PYMES, mientras esta, se encuentre adecuada con las características de ella.

\section{Modelos de comercialización:}

Si bien existen variaciones en cuanto a los modelos de comercialización aplicables por las empresas desde una perspectiva básica son dos las existentes: la venta directa al cliente final y la de venta de empresa a empresa.

\section{Venta Business to Customer}

Este modelo de venta es el más conocido por las PYMES, generalmente enfocadas a la atención de los clientes finales de forma directa. Debido a que la mayoría de los usuarios de internet, con acceso a tarjetas de débito o crédito, han comprado al menos una vez por internet, a medida que las empresas ofrecen sus productos o servicios mediante este, los clientes disponen de mayor libertad y flexibilidad para comparar precios y adquirir productos. En este modelo, disminuye la cadena de suministros, por lo que, tanto empresas como clientes, pueden verse beneficiados. (SOLEDAD, 2014)

Según el estudio sobre venta online en Pyme 2020 mencionado con anterioridad, el 91.5\% de las PYMES que vende por plataforma, lo hace directo al público, debido a la ventaja que supone recibir el dinero de forma directa del cliente y la ganancia no se reparte.(AMVO, 2020)

\section{Venta Business to Business}

Este modelo de ventas es igualmente habitual pero menos constantes en las PYMES, debido a que las transacciones se realizan de empresa a empresa y estas pueden ser de tres tipos:

- Venta única a empresas: Se da cuando los productos por su naturaleza suelen venderse a empresas. Los procesos de venta se ajustan a los procedimientos de las empresas y muy seguramente se deben trabajar con plazos de pago.

- Venta Indistinta a empresas y clientes: Se da cuando la empresa puede cubrir las necesidades de las empresas y los clientes en general.

- Venta diferenciada entre empresas y cliente: Este modelo hace distinción entre precios y cantidades, plazos de pago, entre otras cosas. En general, tienen ofertas y precios distintos para particulares y para empresas. (Empresa Actual, 2020)

De acuerdo con el estudio sobre venta online en México, el 96.6\% de las PYMES, vende por 
este medio a empresas o manejan algún formato mencionado con anterioridad. Esto, debido a la necesidad de apoyo en la distribución de los productos, y les permite ser más rentables a largo plazo por las cantidades que se manejan en este formato de venta. (AMVO, 2020)

Es factible pensar que la base de clientes se amplía cuando una PYME cuenta con las condiciones para comercializar tanto de manera directa al consumidor final como cuando vende a otras empresas por lo que se observa como una buena estrategia que la PYME establezca una estrategia que considere a ambos clientes para no solo incrementar sus ventas sino también diversificarlas y considerando los reportes de la literatura, el uso de los medios digitales debe ser considerado como una relevante opción para basar la estrategia comercial.

\section{MARCO TEÓRICO}

\section{La Mipyme en México}

La definición de una empresa MIPYME en México, está precisada por diferentes aspectos, en la actualidad, incluye a una empresa con capacidad limitada en recursos y trabajadores. Que, acuerdo con el portal de internet del Senado de la república en México, la publicación de la gaceta LXII/3spo-127/54141 (2015), la estratificación de las es de la siguiente forma:

- Una micro se puede encontrar para todos los rectores, por un rango de hasta 10 empleados con un monto de venta anual de hasta 4 millones de pesos.

- Pequeña se encontrará en el sector comercio, industria y servicios, de 11 a 50 empleados de 4 a 100 millones de pesos de venta anual.

- Mediana empresa en sectores de comercio, de 31 a 100 empleados con ventas que van desde 100.01 a 250 millones de peso anuales. Y, en los sectores de servicios e industria, con empleados que van desde los
51 hasta los 250, con ventas anuales desde los 100.01 a 250 millones de pesos.

Con información de INEGI, las PYMES en México representan casi el $99 \%$ de las unidades económicas, que además generan el $80 \%$ de los empleos en México. Existe un estimado de 4 millones de PYMES en México, sin embargo, el $70 \%$ fracasa o cierra sus puertas a $\operatorname{los} 5$ años de apertura.

En Baja California y en Mexicali, el promedio de vida de las empresas es de 7.9 años, siendo el sector del comercio quien tiene más volatilidad.

Aunque la opinión de la causa principal de la falta de crecimiento de las MIPYMES va desde exceso de trámites gubernamentales pasando por competencia de empresas informales, hasta la baja demanda de sus productos.

\section{Canales de distribución adecuados}

Es importante la selección de los canales de distribución, debido a que hay factores determinantes como el precio, que debe estar considerados también por el número de intermediarios que tendrá el producto que se necesita llevar al consumidor final. Por tanto, se entiende que, a más intermediarios, el porcentaje de margen de ganancia se ve disminuido e impacta al precio final del usuario.

Según el libro Canales de distribución y logística (Velazquez Velazquez, 2012) existen 5 diferentes canales principales, el primero de ellos es el que va directo del fabricante al consumidor sin ningún intermediario.

El segundo canal es productor-minoristaconsumidor, como se ve en la Figura 1.1, con este tipo de canal el fabricante vende a una tienda minorista que venden directamente a consumidores. En este punto, hay que considerar la ganancia del minorista en el precio.

El tercer canal es productor-mayoristaminorista-consumidor, en este canal se ven dos niveles, antes de que el consumidor tenga su producto, lo que considera más logística en el movimiento y porcentajes. 
Por último, Fabricantes-agentes-mayoristasminoristas- consumidor, por tanto, se aumenta en tres niveles, lo que produce que los fabricantes o productores se preocupen más por el precio de su producto, debido a que cada nivel lleva su margen de ganancia, y, para muchas empresas PYMES si se considera a la competencia como parte de la fijación de precio.

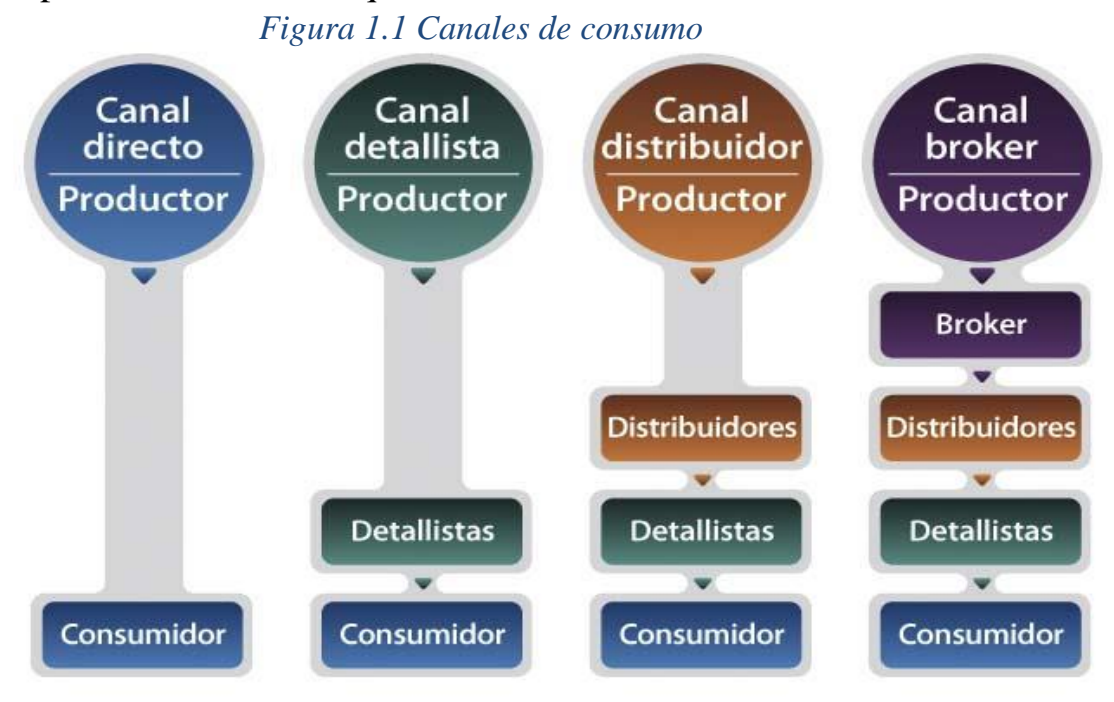

Fuente: Torreblanca, 2018

\section{Comercio electrónico para PYMES}

En México las ventas en línea han tenido un crecimiento del $34 \%$ en el año 2020 con respecto al año anterior. $\mathrm{Y}$, aunque es uno de los canales más atractivos en los últimos años para ventas, no todas las empresas se pueden adaptar con facilidad al e-commerce, sin embargo, hoy por hoy, es una herramienta crucial para despegar en ventas. (Alejandro, 2020)

\section{Estrategias de crecimiento para la empresa}

Los autores Phillip y Milton Kotler ( 2014) en su libro 8 maneras de crecer, desarrollan una serie de estrategias que las empresas pueden utilizar para tener un crecimiento paulatino y constante. En el indican, estrategias como estas:

1. Crecer aumentando la cuota del mercado

2. Crecer fomentando el compromiso de clientes y demás grupos de interés.

3. Crecer desarrollando una marca potente

4. Crecer innovando en productos, servicios y experiencias
5. Crecer a través de la expansión internacional

6. Crecer a través de fusiones, adquisiciones, alianzas y empresas conjuntas.

7. Crecer desarrollando una excelente reputación en responsabilidad social corporativa

8. Crecer mediante colaboraciones con la administración y con algunas ONG.

\section{Atención al cliente en el comercio digital}

De acuerdo con (Moncalvo, 2008) existen cinco principios básicos para dejar de ser un desconocido y estar en un terreno de la desconfianza con el cliente, a pasar a tener una relación de mutuo provecho. Ello es, el respeto, la calidez, el contacto y el seguimiento. Lograr que el cliente sea fiel a una marca es muy difícil, conseguir que el cliente regrese a una plataforma o busque el producto en un multicanal, con todas las opciones del internet, es complicado. Sin embargo, se desarrollan programas de fidelización que tiene éxito en un sistema de incentivos lo cual permite que el cliente adquiera puntajes o niveles y con ello, obtener 
algún beneficio para el interesante. Pudiendo ir desde descuentos por compras determinadas hasta productos gratis.

\section{Indicadores de experiencia en clientes}

Toda empresa debe tener claro que cuidar a los clientes es la base fundamental, las grandes empresas lo saben muy bien y tienen indicadores para ello, sin embargo, no todas las PYMES cuentan con estas herramientas o el conocimiento para medir las experiencias de los clientes, por tanto, se vuelve más difícil su manejo. Según el portal Question Pro (2020), especializado en encuestas e investigaciones de mercado, existen 6 indicadores para medir la experiencia del cliente:

Figure 1.2 Comparación de canales favorables para empresa PYME

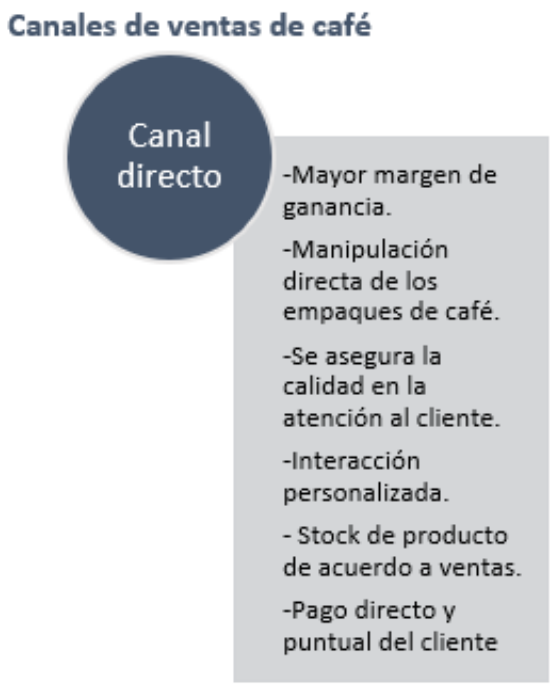

Fuente: Elaboración propia, 2021
- Net Promoter Score (NPS): Ayuda a identificar que tan satisfechos se encuentran los clientes con la marca y si la recomendarían a otras personas, mediante la aplicación de una pregunta que clasifica a los clientes en: detractores, Pasivos y Promotores.

- Indicador del esfuerzo del cliente (CES): recolecta información acerca de los factores que pueden propiciar negativamente al cliente. Se clasifican seleccionando opciones como "muy difícil" a "Muy fácil"

- Puntuación de Satisfacción del cliente (CSAT): En este método se aplican preguntas que van desde "Muy satisfecho" hasta "Muy insatisfecho".

- Valor de vida del cliente (CLV): En este determinan cual es la ganancia que un

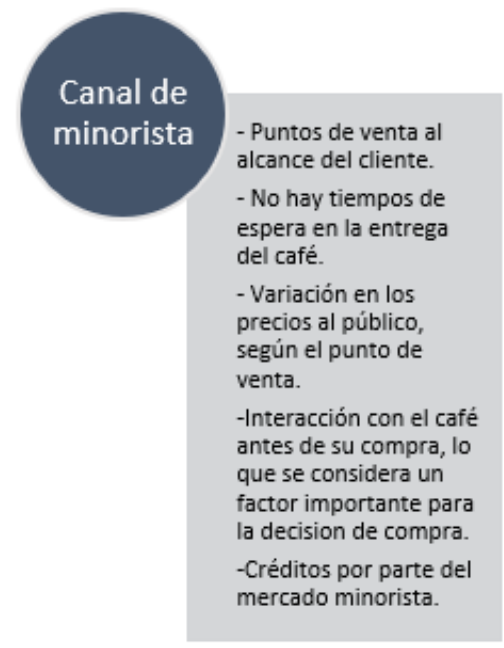

cliente deja a una marca según sus niveles.

- Tasa de respuesta y solución de problemas: Este mide el tiempo que tarda el equipo de la empresa para responder a los clientes y solucionarle sus problemas.

- Tasas de abandono y retención del cliente: En este se conocen los motivos por los cuales los clientes no están contentos con la marca y buscan evitar la rotación de los mismos.

Estas herramientas aportan a las PYMES información invaluable, con ello, no solamente estarían conociendo a sus clientes, si no sabrían si el servicio y los productos que manejan son satisfactorios para ellos.

Lo ideal, conforme a la lectura realizada, es la comercialización con el menor número de intermediarios posibles para una 
empresa pequeña, es por ello, que se analizan dos diferentes formas de comercializar productos, en la figura 1.2, se observan las ventajas de los dos tipos de canales favorables, El canal directo, permite mayor margen de ganancia, manipulación y control de stock de producto, se asegura la calidad en la atención al cliente, pago directo y puntual por parte del cliente. Por el contrario, el canal minorista o de cadenas comerciales permite puntos de venta al alcance inmediato del cliente, no existe tiempo de espera, el porcentaje de venta.

\section{MÉTODO}

Esta investigación tiene un enfoque cualitativo, se revisó diversa bibliografía referente a las PYMES en México, así como infográficos, videos, documentos, paginas web, entre otros.

Asimismo, mediante la triangulación; entiéndase por triangulación como "verificación de la existencia de determinados fenómenos y la veracidad de las explicaciones individuales mediante la recolección de datos de una serie de informantes y una serie de fuentes, para posteriormente comprar $y$ contrastar una explicación con otra, con el fin de elaborar un estudio lo más equilibrado posible" (Open University Couse E811,1988,citado por Bell, 2020), se encontrarán los factores claves para las PYMES dedicadas a la comercialización y venta del café, fuera del mercado retail.

Además, reúne elementos y características de un enfoque descriptivo; "Se entiende como el acto de representar por medio de palabras las características de un fenómeno, hechos, situaciones, cosas, personas y demás seres vicos, de tal manera que quien lea o interprete los evoque en la mente". (Niño Rojas, 2021)

\section{RESULTADO}

Después de las valoraciones y análisis realizados a partir del marco teórico, se establecen las recomendaciones siguientes: Los resultados que se presentan, derivan de los factores claves de las PYMES dedicadas a la venta de café en retail, sin embargo, esta evaluación determina 5 estrategias con mayor impacto:

- Estrategia efectiva de ventas, para plataformas digitales

- Realización de campaña de relaciones publicas y mercadotecnia para mantener alta valoración en clientes

- Utilizar las herramientas digitales disponibles para mejorar venta.

- Búsqueda de créditos con proveedores para facilidades de pago.

Por otro lado, los canales de comercialización seleccionados para la empresa se realizan con base a la demanda. Con ello, se verifica que la distribución se realice de acuerdo al tipo de cliente y sus necesidades de consumo. Con referencia al documento de la OCDE, (2021) el análisis enmarca que la venta de café por el canal directo, tiene estas ventajas competitivas genera mayor margen de ganancia, una manipulación directa de los empaques de café, se asegura la calidad en la atención al cliente, interacción personalizada, stock de producto de acuerdo a ventas, pago directo y puntual del cliente.

Con lo anterior, la recomendación principal va en tres principales vías, buscar las bases digitales necesarias para desarrollar la venta directa al público, buscar financiamiento directo de proveedores, crear estrategias de venta para las bases digitales adecuadas.

\section{CONCLUSIONES}

Como se ha mencionado en el marco conceptual, la transformación a las ventas digitales para toda empresa es importante; en el caso de las PYMES, les genera un impacto altamente positivo para poder seguir creciendo.

Para que una empresa dedicada al rubro de la comercialización del café, siendo una pyme, migre de una comercialización física a un apoyo con la comercialización digital, es importante que trabaje en un plan de 
capacitación y networking, sobre todo en aquellos temas que sean de estrategia en ventas y de atención al cliente en páginas web. Por otro lado, crear una estrategia de entrega y logística, debido a la demanda que puedan tener, la claridad con la que aborden los pedidos y entregas será crucial para su crecimiento en el enfoque. 


\section{REFERENCIAS}

Alejandro, C. (2020, August 18). Ecommerce: Un game-changer para las compañías mexicanas. Revista

Merca2.0. https://www.merca20.com/camino-a-la-recuperacion-como-el-ecommerce-ha-ayudado-apymes-mexicanas-a-sobrevivir/

AMVO, A. M. de venta online. (2020). Estudio sobre Venta Online en PyMEs 2020. Asociación Mexicana de venta online.

Bell, J. (2020). Lea Cómo hacer tu primer trabajo de investigación. Gedisa.

https://es.scribd.com/book/469352843/Como-hacer-tu-primer-trabajo-de-investigacion-Guia-parainvestigadores-en-educacion-y-ciencias-sociales

Empresa Actual. (2020, January 27). Modelos de negocio e-commerce: B2B, B2C, C2B ¿.. ¿Qué tipos de comercio electrónico existen? Empresa Actual. https://www.empresaactual.com/tipos-comercioelectronico/

Guru. (n.d.). Marketing digital para pymes.

IAB México. (2019, October 4). PANORAMA DEL COMERCIO ELECTRÓNICO EN MÉXICO 2019-IAB México. https://www.iabmexico.com/estudios/infografia-panorama-del-comercio-electronico-enmexico-2019/

Kotler, P., \& Kotler, M. (2014). 8 Maneras de crecer. BPR Business Publishers Roundtable. https://es.scribd.com/book/297503971/8-Maneras-de-crecer

Moncalvo, A. (2008). Comercio electrónico para pymes (1st ed.). LECTORUM-UGERMAN de

\section{LECTORUM S.A.}

Niño Rojas, V. M. (2021). Lea Metodología de la investigación de Víctor Miguel Niño Rojas en línea| Libros. https://es.scribd.com/book/494173719/Metodologia-de-la-investigacion-Diseno-ejecucion-einforme-2\% C2\%AA-Edicion

OECD. (2021). The Digital Transformation of SMEs | READ online. http://www.keepeek.com/Digital-AssetManagement/oecd/industry-and-services/the-digital-transformation-of-smes_bdb9256a-en

Question Pro. (2020, September 21). Indicadores para medir la experiencia del cliente. QuestionPro. https://www.questionpro.com/blog/es/indicadores-para-medir-la-experiencia-del-cliente/

Sánchez, S. (2020, October 6). Nespresso quintuplica ventas en línea por consumo en hogares mexicanos • Negocios • Forbes México. Forbes México. https://www.forbes.com.mx/negocio-nespressoquintuplica-ventas-en-linea-por-consumo-en-hogares-mexicanos/

Senado de la república. (2015, Abril). Senado de la República. https://www.senado.gob.mx/64/gaceta_del_senado/documento/54141

SOLEDAD, C. F. (2014). Venta online. Ediciones Paraninfo, S.A.

Torreblanca, F. (2018, July 24). Niveles de los canales de distribución: Canal directo y canal indirecto. Blog de Francisco Torreblanca. https://franciscotorreblanca.es/niveles-de-los-canales-de-distribucion/

Velazquez Velazquez, E. (2012). Canales de distribución y logística (Primera). Red Tercer Milenio. 